$65 / 577 ; \mathrm{p}<0.001)$ The cumulative survival rate of the non-pericardial effusion group was 96.9\%, 95.8\%, 93.6\%, and $87.1 \%$ at 1 year, 2 years, 5 years and 10 years; which were $89.0 \%$, $86.0 \%, 78.4 \%$, and $69.6 \%$, respectively, in the pericardial effusion group.

Conclusions This study underlines the prevalence and outcome of pericardial

effusion/cardiac tamponade in SLE, which is significant related to patient survival.

\section{LATE-ONSET SYSTEMIC LUPUS ERYTHEMATOSUS IN CHINESE PATIENTS REVEALS A NON-BENIGN DISEASE}

Z Chen*, GS Wang, XP Li, XM Li. Affiliated Anhui Provincial Hospital - Anhui Medical University, Rheumatology and Immunology, Hefei, China

\subsection{6/lupus-2017-000215.433}

Background and aims To assess clinical manifestations, laboratory findings and outcome of a cohort of Chinese SLE patients with onset at age $\geq 50$ from a tertiary referral centre by performing a case control study.

Methods All hospitalised SLE patients in last five years were retrospectively reviewed. Patients who developed disease at or after the age of 50 were considered as LOSLE. 1:1 matched control SLE patients, admitted in the same period with initial symptoms onset before the age of 50, paired by race and disease duration, were randomly selected. Clinical manifestations, laboratory findings, therapies as well as outcome of the two groups of patients were compared.

Results LOSLE amounts to $4.3 \%(35 / 814)$ of all SLE patients in our centre. The most frequent clinical manifestations of LOSLE were arthralgia (60\%), fever (37.1\%) and serositis $(37.1 \%)$. Compared with younger onset patients, LOSLE had less incidence of rash, oral ulcers, Raynaud's phenomenon, but had more renal involvement and secondary sjogren's syndrome. In laboratory findings, positive anti-dsDNA, anti-Sm, anti-SSA, anti-SSB and anti-RNP antibody were less frequent in LOSLE compared with younger SLE patients. Additionally, SLEDAI was lower in LOSLE group at diagnosis and fewer LOSLE patients received cytotoxic agents. However, one -year survival rate was lower in LOSLE group than control patients (78\% and 91\%, respectively).

Conclusions Patients of LOSLE tend to be milder and atypical in symptoms, signs and laboratory findings, but they are prone to experience more severe renal damage and higher mortality. LOSLE appear to be a non-benign disease in our cohort of Chinese patients.

\section{GOING DOWN A DIFFERENT PATH": A QUALITATIVE EXPLORATION OF COSTS INCURRED BY CANADIAN PATIENTS WITH SYSTEMIC LUPUS ERYTHEMATOSUS (SLE)}

IJ Dixon, 'S Elliott, ${ }^{2} \mathrm{~S}$ Pike, ${ }^{3} \mathrm{C}$ Seger* ${ }^{3} \mathrm{R}$ Church, ${ }^{4} \mathrm{~A}$ Clarke. ${ }^{1}$ University of Waterloo, Geography and Environmental Management, Waterloo, Canada; ${ }^{2}$ McGill University, Montreal, Canada; ${ }^{3}$ Lupus Society of Alberta, Calgary, Canada; ${ }^{4}$ University of Calgary, Department of Medicine Division of Rheumatology Cumming School of Medicine, Calgary, Canada

10.1136/lupus-2017-000215.434

Background and aims Prior investigations into the economic costs of Systemic Lupus Erythematosus (SLE) among affected individuals have primarily been quantitative and focused only on direct costs. Given that SLE predominantly impacts patients during their prime working years and symptoms can be complex, this research employs a qualitative approach to explore and contextualise the direct as well as indirect costs incurred by Canadian patients with SLE.

Methods Semi structured in-depth interviews were conducted with 8 key informants (3 expert physicians, 5 representatives from patient advocacy groups) and 28 adult SLE patients. Interviews were audio recorded, transcribed verbatim and coded for recurring themes.

Results Findings reveal that the early manifestations of the disease significantly rerouted the course of participants' economic livelihoods, often to part-time precarious employment and in some cases forced them to leave the workforce all together. A lack of health insurance benefits and poor health coverage for complementary care created additional expenses. Openness about diagnosis within the workplace varied greatly amongst participants, though all noted the challenges created by a general lack of information and public awareness about SLE. The use of workplace accommodations was met with mixed success and greatly depended on the work setting.

Conclusions Changes within Canadian policy could improve access to resources for promoting healthy lifestyles and disease management for those with SLE which, in turn, would serve to enhance economic security. This research adds deeper understanding to the widespread and multifaceted costs associated with SLE, elucidating the long-term consequences of SLE and its implications for social policy.

\section{\begin{tabular}{l|l}
435 & DISEASE ACTIVITY PATTERNS AMONG FILIPINO
\end{tabular} PATIENTS WITH SYSTEMIC LUPUS ERYTHEMATOSUS: A 3-YEAR OBSERVATIONAL STUDY}

MFJ Edar, L Zamora, S Navarra. University of Santo Tomas, Internal Medicine, Manila, Philippines

\subsection{6/lupus-2017-000215.435}

Background and aims To describe organ involvement, disease activity patterns, and damage characteristics in a cohort of Filipino patients with systemic lupus erythematosus (SLE), observed over 3 years.

Methods Consenting SLE patients at University of Santo Tomas (UST) Hospital with minimum 3 visits/year were assessed over 3 years until July 2016. Disease activity patterns included clinically quiescent disease (CQD; SLEDAI $=0$ in 3 visits); minimal persistent disease (MDA; SLEDAI $=1$ in $>1$ visit); relapsing remitting disease (RRD; SLEDAI $\geq 2$ in 1 of 3 visits); chronic active disease (CAD; SLEDAI $\geq 2$ in at least 2 of 3 visits). SLICC Damage Index (SDI) characteristics were correlated with steroid use.

Results 127 SLE patients (117, 92\% females), mean 28.81 +10.14 SD (range 4-57) age at diagnosis, mean $7.94+5.61$ SD (range 1-25) years disease duration. Organ involvement included mucocutaneous (121,95\%), musculoskeletal (113, $89 \%)$ and renal $(87,68.5 \%)$. At 1 year there were 48 (40.7\%) patients with CAD, 37 (31.4\%) CQD, 30 (25.4\%) RRD, 3 (2.5\%) MDA. At 3 years, CAD patients totaled 23 (19.5\%), CQD 59 (50\%). Table 1 Mean cumulative prednisone dose was $21.42+17.63$ at initial damage involvement. Cataract was most common SDI (26, 20.5\%), significantly correlated with cumulative steroid, $\mathrm{p}<0.01$. There were 131 